\title{
Promising Advances in Budd-Chiari Syndrome in Children
}

\author{
VANitha VASudevan ${ }^{1}$ ANd Devendra Chandra Joshi ${ }^{2}$ \\ From ${ }^{1}$ Department of General Surgery and Surgical Oncology, Palmetto General Hospital, and ${ }^{2}$ Department of General and

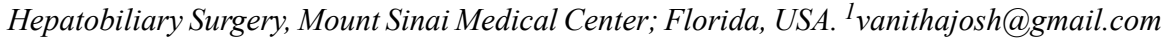

I was with great interest that we read the article by Redkar, et al. [1] on pediatric Budd-Chiari syndrome (BCS) and the various therapeutic interventions. BCS is a rare clinical syndrome in children, estimated to account for $7.4 \%$ of pediatric chronic liver disease in India [2]. Pediatric cases accounted for about $21 \%$ of the cases of BCS in a large Indian case series [3]. Standardized diagnostic and management protocols are lacking in pediatric literature.

As noted in the article by Redkar, et al. [1], inherited hypercoagulable states like Protein $\mathrm{C}$ and $\mathrm{S}$ deficiency are the most common causes for BCS in children; however, Western data show predominance of hematological disorders such as polycythemia vera and essential thrombocytosis [4,5]. One series from India showed dehydration secondary to diarrhea and fever, preceding BCS in $45 \%$ of the cases [5]. Detailed work-up was not available in most retrospective series; however, we believe that this should be an essential component in diagnostic work-up as certain genetic prothrombotic states can be managed medically (interferon, JAK2 inhibitors, stem cell transplantation) [4].

In pediatric population, presentation of $\mathrm{BCS}$ is mostly chronic $[5,6]$. Clinical presentation with abdominal distension secondary to ascites, with Serum ascites-albumin gradient $(\mathrm{SAAG})>1.1$, should alert to the presence of Budd-Chiari Syndrome. This is especially important in countries with predominance of tuberculosis, where ascitic fluid with SAAG $<1.1$ denotes a peritoneal cause for ascites [5]. As rightly pointed out by the authors, high degree of suspicion and early diagnosis is the key.

Untreated BCS has a poor prognosis with death resulting from progressive liver failure in 3 months to 3 years from diagnosis [7]. Various management algorithms have been described in adult literature. Plessier's algorithm, which includes medical management, recanalization, Transjugular intrahepatic portosystemic shunt (TIPS) and liver transplant has been successfully shown to have 5-year survival of $89 \%$ in adult population [8]. Surgical shunts are not a part of this algorithm.

Large series studying therapeutic interventions for BCS are sparse in pediatric literature. This would make the current article [1] one of the few published case series on management of BCS, most of them being from the Indian subcontinent. This past decade has seen a rise in early and aggressive use of percutaneous recanalization techniques for hepatic venous decompression $[5,6,9,10]$. Hepatic vein is the most common site of obstruction noted $[5,6]$. The exact interventional technique depends on the hepatic venous anatomy, exact site and extent of venous outflow obstruction, caliber of the vessels involved, and also the technical expertise available.

Few prior case series on BCS are worth examination. Nagral, et al. [5] described 16 children who were treated with angioplasty (for vein $\leq 4 \mathrm{~mm}$ ), hepatic vein stenting (for vein $\geq 5 \mathrm{~mm}$ ) and TIPS (if all hepatic veins were occluded). At a median follow-up of 31 months, they reported least patency with angioplasty (25\%) and higher with stenting and TIPS. Kathuria, et al. [6] also reported safety and technical feasibility of radiological interventional techniques in children with BCS with use of hepatic stenting and TIPS with 75\% patency recorded at a short-term (6.5-month) follow-up. Sharma, et al. [9] reported outcomes at long-term median follow up of 44 months. Angioplasty had success rates of only $43 \%$ while stenting and TIPS were successful in $66 \%$ and $73 \%$, respectively. They were able to preserve liver function and delay need for transplantation. Another series from China [10] also reported the successful use of angioplasty with documented significant drop in hepatic venous pressure, with a $30 \%$ restenosis rate at 25 months follow-up, most of whom were amenable for redo angioplasty.

The current article is interesting because it describes early and aggressive use of angioplasty with excellent success rates, and also advocates early referral for surgical shunt or liver transplantation, based on presence 
or absence of liver cirrhosis. Although it appears that they have a one-year follow up, the exact anticoagulation and imaging follow-up protocols have not been detailed. It is also unclear if any repeat angioplasty was attempted prior to surgical referral. These details may be crucial if we want to develop therapeutic protocols. Hepatic large balloon venous angioplasty has been noted, in a large adult series, to have excellent long-term patency rates [11]. However, the vessel caliber in pediatric population may be a limitation to wider use of angioplasty, as with hepatic vein stenting.

It is very promising to see aggressive and early use of radiological interventional techniques for hepatic venous outflow obstruction. Since there seems to be a relative abundance of cases of BCS in India and also increased technical expertise, it would be the next right step to perform multicenter prospective studies in India with the goal of establishing diagnostic and therapeutic manage-ment protocols for pediatric BCS, based on the data already available from the above studies.

Funding: None; Competing interest: None stated.

\section{REFERENCES}

1. Redkar R, Bangar A, Hathiramani V, Raj V, Swathi C. Pediatric Budd-Chiari syndrome: A case series. Indian Pediatr. 2018;55:871-3.

2. Alam S, Khanna R, Mukund A. Clinical and prothrombotic profile of hepatic vein outflow tract obstruction. Indian J Pediatr. 2014;81:434-40.

3. Dilawari JB, Bambery P, Chawla Y, Kaur U, Bhusnurmath SR, Malhotra HS, et al. Hepatic outflow obstruction (Budd-
Chiari syndrome). Experience with 177 patients and a review of the literature. Medicine (Baltimore). 1994;73: 21-36.

4. Nobre S, Khanna R, Bab N, Kyrana E, Height S, Karani J, et al. Primary Budd-Chiari syndrome in children: King's College Hospital experience. J Pediatr Gastroenterol Nutr. 2017;65:93-6.

5. Nagral A, Hasija R, Marar S, Nabi F. Budd-Chiari syndrome in children: experience with therapeutic radiological intervention. J Pediatr Gastroenterol Nutr. 2010;50:74-8.

6. Kathuria R, Srivastava A, Yachha SK, Poddar U, Baijal SS. Budd-Chiari syndrome in children: clinical features, percutaneous radiological intervention, and outcome. Eur J Gastroenterol Hepatol. 2014;26:1030-8.

7. Horton JD, San Miguel FL, Membreno F, Wright F, Paima J, Foster P, et al. Budd-Chiari syndrome: illustrated review of current management. Liver Int. 2008;28:45566.

8. Plessier A, Sibert A, Consigny Y, Hakime A, Zappa M, Denninger $\mathrm{MH}$, et al. Aiming at minimal invasiveness as a therapeutic strategy for Budd-Chiari syndrome. Hepatology. 2006;44:1308-16.

9. Sharma VK, Ranade PR, Marar S, Nabi F, Nagral A. Long-term clinical outcome of Budd-Chiari syndrome in children after radiological intervention. Eur J Gastroenterol Hepatol. 2016;28:567-75.

10. Wang L, Zu MH, Gu YM, Xu H, Zhang QQ, Wei N, et al. [Budd-Chiari syndrome in children and adolescents: therapeutic radiological intervention]. Article in Chinese. Zhonghua Er Ke Za Zhi. 2013;51:590-4.

11. Ding PX, Zhang SJ, Li Z, Fu MT, Hua ZH, Zhang WG. Long-term safety and outcome of percutaneous transhepatic venous balloon angioplasty for Budd-Chiari syndrome. J Gastroenterol Hepatol. 2016;31:222-8. 